\title{
Sensorimotor deficits remain despite resolution of symptoms using conservative treatment in patients with tennis elbow: A randomized controlled trial
}

\section{Leanne M. Bisset, PhD, Michel W. Coppieters, PhD, Bill Vicenzino, PhD}

Sensorimotor deficits remain despite resolution of symptoms using conservative treatment in patients with tennis elbow: a randomized controlled trial. Arch Phys Med Rehabil 2009;90: 1-8.

\begin{abstract}
Objectives: To evaluate the time course of sensorimotor deficits in lateral epicondylalgia (LE; tennis elbow) and how these deficits may be influenced by common conservative treatments.

Design: Single-blind randomized controlled trial.

Setting: University laboratory, Australia.

Participants: A total of 198 participants with unilateral LE of a minimum 6 weeks duration and normative data from a group of 40 healthy participants.
\end{abstract}

Interventions: Participants with LE were randomly assigned to physical therapy, corticosteroid injection, or a wait-and-see approach with ergonomic advice.

Main Outcome Measures: Upper-limb reaction time (simple, 1-choice and 2-choice reaction time) and speed of movement were assessed at baseline and 6 and 52 weeks of follow-up.

Results: Patients with LE exhibited delayed reaction times $(P<.001)$ and slower movement speeds $(P<.001)$ for both the affected and unaffected arm at baseline, compared with normative data.

Despite some improvement over time, significant bilateral deficits remained compared with healthy participants, with no significant differences between the affected and unaffected arm. Overall, there was no significant difference between the 3 treatment groups over time.

Conclusions: Sensorimotor deficits remain relatively unchanged over time and are present bilaterally in patients with unilateral LE. Treatment did not influence the time course of the sensorimotor deficits. This prompts conjecture as to the relationship and clinical relevance of sensorimotor deficits in LE. It should be noted that treatment evaluated in this study did not specifically target sensorimotor function, and further investigation into the significance of sensorimotor deficits in patients with LE is required.

Key Words: Sensorimotor; Reaction time; Rehabilitation; Tennis elbow.

Lateral epicondylalgia (tennis elbow) is primarily a condition of pain in the area of the lateral humeral epicondyle. LE affects $1 \%$ to $3 \%$ of the general population and up to $15 \%$ of workers in atrisk industries. ${ }^{1-4}$ In addition to the clinical characteristics of pain and reduced function, ${ }^{5-7}$ bilateral sensorimotor impairments are present in patients with LE. ${ }^{8,9}$ The sensorimotor system is defined as a component of the motor control system and is used to describe the sensory, motor, and central integration and processing components involved in maintaining stability during dynamic movement. ${ }^{10,11}$ The sensorimotor system includes joint position sense, sensation of force, and neuromuscular control that involves both feedforward and feedback mechanisms. ${ }^{11}$ Reaction time and speed of movement are simple measures of sensorimotor function and have been shown to be significantly slower in participants with LE compared with healthy controls. ${ }^{8,9}$ Deficits in sensorimotor function have been identified previously in other chronic musculoskeletal conditions such as patellofemoral pain syndrome, ${ }^{12}$ low back pain, ${ }^{13,14}$ and shoulder disorders. ${ }^{15,16}$

Conservative treatments have been found to be beneficial in improving sensorimotor function in knee and shoulder disorders ${ }^{17-19}$; however, the impact of conservative treatments on sensorimotor deficits in LE is unknown. Typically, conservative treatment is aimed at reducing pain and improving function in patients with LE. There is strong evidence to suggest that most patients with LE report improvement in their condition in the long term (52 weeks) if they adopt a wait-and-see approach, a physical therapy program, or corticosteroid injections. ${ }^{20-22}$ In particular, a physical 
therapy program of mobilization with movement and therapeutic exercise was found to be more effective than a wait-and-see approach in the short term and superior to corticosteroid injections in the long term. Corticosteroid injections were found to be superior to both physical therapy and waitand-see in the short term, but had a high recurrence rate after 6 weeks. ${ }^{20-22}$ The effect of these treatment approaches, while demonstrating efficacy on outcome measures of pain and function, is unknown for sensorimotor impairments in patients with LE.

The aim of this study was to evaluate the effect of physical therapy and corticosteroid injections compared with a wait-and-see (natural history) approach on sensorimotor function. We hypothesized that sensorimotor deficits would abate over time, in line with the previously reported resolution of the condition, ${ }^{20}$ and that an active physical therapy treatment would speed up this recovery more than a corticosteroid injection or natural resolution over time.

\section{METHODS}

We conducted an assessor-blinded, randomized controlled trial that investigated the effects of 3 treatment interventions for patients with LE. ${ }^{20}$ Participant recruitment, determination of eligibility, and the randomization procedure have been described elsewhere, as have the primary outcome measures of global improvement, assessor severity, and pain-free grip strength. ${ }^{20}$ In addition to these primary outcome measures, we evaluated sensorimotor function, which is the focus of this article. All participants gave written informed consent prior to enrolment into the study, and ethical approval was gained from the institutional human research ethics committee prior to commencement of data collection.

\section{Participants}

One hundred ninety-eight participants, recruited from the general community (Brisbane, Australia), age 18 to 65 years with a clinical diagnosis of unilateral LE of a minimum 6 weeks duration were enrolled in the trial. A prerequisite for inclusion was that participants had not received any active treatment by a health practitioner in the previous 6 months. The participants were randomly assigned via concealed allocation to physical therapy, corticosteroid injections, or a wait-and-see approach. Demographic and baseline characteristics are reported in table 1, and figure 1 illustrates the flow of participants through the trial.

\section{Intervention}

The participants assigned to the wait-and-see group were given ergonomic advice on how to modify their daily activities to avoid aggravating their elbow symptoms. Participants assigned to the corticosteroid injection group were given a local injection of $1 \mathrm{~mL} 1 \%$ lignocaine with $10 \mathrm{mg}$ of triamcinolone acetonide in $1 \mathrm{~mL}$, at painful points of the elbow, with the advice to return gradually to normal activities. The physical therapy treatment consisted of Mulligan's Mobilization with Movement manipulation technique ${ }^{23}$ and a customized therapeutic exercise program. ${ }^{24}$ There were 8 treatment sessions over 6 weeks. Participants in the physical therapy group were also instructed on a home exercise program and self-mobilization.

\section{Outcome Measures}

A series of reaction time tasks for the upper limb were evaluated by a blinded assessor using a Sensorimotor Interface Hand Module. ${ }^{\text {a }}$ Reliability has previously been reported for this module. ${ }^{25}$ The tasks consisted of SRT, 1-choice reaction time and corresponding speed of movement 1, and 2choice reaction time and corresponding speed of movement 2 . Reaction times were measured in milliseconds as the time delay between a light stimulus and the release of the hand from the central plate (figure 2). In the simple reaction task, participants were asked to move their hand off the plate in reaction to the light. The other 2 reaction time tests required hand movements from the central plate toward the plate with a light stimulus, with either 1-choice reaction time or 2-choice reaction time possible choices. Movement speed $(\mathrm{cm} / \mathrm{s})$ was calculated by dividing the distance between the 
central and target plate by the time taken for hand movement from 1 plate to the other. All participants were tested according to the protocol provided by the manufacturer. ${ }^{26}$ The tasks were assessed in a predetermined sequence-SRT, 1-choice reaction time + speed of movement 1, 2choice reaction time + speed of movement 2-with the unaffected side measured first to facilitate

familiarization with the protocol. ${ }^{27}$ Outcome measures were taken at baseline and at 3, 6, 12, 26, and 52 weeks of follow-up to evaluate the time course of sensorimotor function. In addition to the evaluation of changes over time, the results from the participants with LE were compared with previously published normative data. ${ }^{9}$

\section{Data Analysis}

Baseline characteristics known to affect upper-limb reaction time and speed of movement (age, sex, hand dominance, duration of condition $)^{25}$ were evaluated for the pooled LE cohort using 2-way analysis of variance $(P<.05)$. The primary end points for the trial were 6 weeks (short-term) and 52 weeks (long-term). For the LE population, we derived estimates of effect for all continuous outcome measures by using change scores from baseline in a 3-way analysis of variance with time, treatment group, and side (affected, unaffected) defined as fixed factors. We reported adjusted results if patient characteristics (eg, age, sex) were found to influence outcomes over time significantly. Significance was set at $0.01(99 \%$ CI) to compensate for the increase in type I error resulting from multiple testing. In addition, comparison between the LE group at baseline with the previously reported control group data, ${ }^{9}$ and the LE group at 52 weeks of follow-up with the control group data $(\mathrm{n}=40)$, were performed using a 2-way analysis of variance with time (baseline and 52 weeks) and condition (LE and normative) as fixed factors. For these post hoc comparisons, significance was set at .01. All statistical analyses were performed on an intention-to-treat basis. In addition to statistical significance, whether the size of the differences were clinically meaningful was judged using a data-driven approach in which previously reported standard error of the measure acted as a proxy for the minimal clinically important difference. ${ }^{28}$

\section{RESULTS}

\section{Demographic Effects}

There were differences in baseline sensorimotor measures based on sex, arm dominance, age, and duration of the condition at the time of presentation. Men were significantly faster than women for SRT (mean difference of $11 \mathrm{~ms}$ for their affected arm, 95\% CI, 1-20; and 10ms for their unaffected arm, 95\% CI, $0.6-19$ ), speed of movement 1 (mean difference affected arm, 13cm/s, 95\% CI, 6-20; unaffected arm, $11 \mathrm{~cm} / \mathrm{s}, 95 \% \mathrm{CI}, 5-17$ ), and speed of movement 2 (mean difference affected arm, $15 \mathrm{~cm} / \mathrm{s}, 95 \%$ CI, 8-23; unaffected arm, $11 \mathrm{~cm} / \mathrm{s}, 95 \%$ CI, 4-19) (fig 3). The dominant side affected was significantly slower than the nondominant side at baseline for 1-choice reaction time (mean difference, $14 \mathrm{~ms}, 95 \% \mathrm{CI}, 2-26$ ) and 2-choice reaction time (mean difference, $15 \mathrm{~ms}, 95 \%$ CI, 227). In contrast, the dominant side affected was significantly faster than the nondominant side affected for speed of movement 2 by $9 \mathrm{~cm} / \mathrm{s}$ (95\% CI, 0.3-18) (fig 4). There was a significant difference in reaction times between older and younger participants for the baseline measures of affected 2-choice reaction time and unaffected SRT (fig 5). To understand better the influence of age on these measures, we subgrouped our participants according to decades $(<40 \mathrm{y}, 41-50 \mathrm{y}$, $>51 \mathrm{y})^{25}$ and found the youngest group $(<40 \mathrm{y})$ was significantly faster for affected side 2 -choice reaction time (mean difference, $18 \mathrm{~ms}, 95 \% \mathrm{CI}, 1-34$ ) and slower for unaffected side SRT (mean difference, $16 \mathrm{~ms}, 95 \% \mathrm{CI}, 3-28$ ) compared with the oldest age group ( $>50 \mathrm{y}$ ). For duration of condition (fig 6), participants with a longer duration of condition at presentation (split about the median of 22 weeks) were significantly faster on SRT than those with a shorter duration of condition (mean difference, $11 \mathrm{~ms}, 95 \%$ CI, 1-20).

\section{Comparison With Normative Data}


All measures of reaction time and speed of movement were significantly delayed in the LE group at baseline compared with normative data $(P<.001)$ (see table 2; figs $7-9)$. Despite a tendency towards improvement in reaction time, the LE group did not significantly improve from baseline to 6 and 52 weeks of follow-up (see table 3). Furthermore, the LE group remained significantly delayed in reaction times $(P<.001)$ and slower in speeds of movement $(P<.001)$ at 52 weeks compared with normative data (see table 2; figs 7-9). There was no significant difference in sensorimotor performance between affected and unaffected sides at follow-up in the LE cohort $(P>.30)$ (see table $3)$.

\section{Between-Group Effects}

The sensorimotor measures were similar at baseline between all 3 treatment groups (wait-and-see, corticosteroid injection, and physical therapy) within the LE cohort (see table 3). The type of treatment did not significantly influence sensorimotor performance over time for any measure (see table 3).

\section{DISCUSSION}

Sensorimotor deficits such as delayed upper-limb reaction time and slower speed of movement are present in participants with LE. This study is the first to reveal that these deficits do not return to values comparable to those of healthy controls over a 12- month period in participants with LE. Reaction times showed a tendency toward normalization over the first 6 weeks, but this change slowed after 6 weeks, and a plateau was observed for the remainder of the follow-up. This pattern of improvement in the first 6 weeks was not significant for reaction time or speed of movement measures. The plateau in improvement seen after 6 weeks and the deficits noted at long-term follow-up when the LE group data were compared with normative data, suggest that sensorimotor deficits do not recover in the long term. Overall, the affected and unaffected side behaved similarly for all measures, with no significant difference between sides over time, suggesting that the bilateral deficits previously identified ${ }^{8,9}$ persist over time in patients with a unilateral condition.

The sensorimotor deficits identified in participants with LE may be explained through changes to central neuromotor processing. There is evidence to suggest that pain has an effect on timing of muscle activation and reorganization within the primary somatosensory cortex of the brain. ${ }^{29,30}$ It may be that with continued use of the affected limb in the presence of pain, such as that experienced with LE, movement of the upper limb is altered. ${ }^{31,32}$ Faulty proprioceptive input through altered movement may then disrupt the internal representation of the body that the brain uses to control movement. ${ }^{33}$ This disruption may cause incongruence between motor output and sensory feedback, thereby resulting in impairments in sensorimotor function. ${ }^{34}$ In this study, however, measures of sensorimotor function did not follow a pattern of recovery similar to that of measures of pain and function. Measures of pain and function do not appear to capture directly the sensorimotor deficits in participants with LE, and therefore, there is a need to investigate further the significance and relevance of sensorimotor deficits in LE.

Central neuromotor changes and communication between cerebral hemispheres ${ }^{35,36}$ may be responsible for the bilateral deficits in upper-limb reaction time and speed of movement seen in this study as well as previously. ${ }^{8,9}$ It has been proposed that transfer of information between homologous cortical areas during a unilateral motor task occurs in the healthy population, regardless of hemisphere dominance. ${ }^{35,36}$ Perhaps through a mechanism of interhemispheric communication, ${ }^{36}$ the impaired motor task on the injured side is mapped onto the uninjured side, resulting in bilateral deficits associated with this group of participants with unilateral LE. Baseline measures of sensorimotor function were different between sexes, dominant/nondominant sides, ages, and duration of condition. These findings are in line with previously reported data ${ }^{25}$ with respect to sex, in which men demonstrated significantly faster speed of movement and reaction time, but contrast with a previous study on the influence of the dominant side and age on sensorimotor measures. 
Kauranen and Vanharanta, ${ }^{25}$ as part of a reliability study, reported standard error of the measures for 2-choice reaction time $(17.4 \mathrm{~ms})$ and speed of movement $2(8.8 \mathrm{~cm} / \mathrm{s})$ for the upper limb. Using the assumption that a minimal clinically important difference will lie outside the standard error of the measure, ${ }^{28}$ it is possible that the differences seen between subgroups of patients at baseline, although statistically significant, are not clinically meaningful, particularly for the reaction time measures, where the differences averaged 13.5 milliseconds. Alternatively, these differences between our data and previously reported data may be a function of the condition of LE, because the previous study used a healthy uninjured cohort. ${ }^{25}$

The physical therapy treatment in this study, which included pain-free upper-limb endurance and strength exercises, did not preferentially influence the sensorimotor deficits as hypothesized. The effects of a 10-week exercise program on sensorimotor function in 16 healthy women has been investigated by Kauranen et $\mathrm{al}^{37}$ in a previous study that reported a statistically significant improvement of $16 \mathrm{~ms}$ in 2-choice reaction time, but no significant change in speed of movement 2, pre-exercise to postexercise. The authors postulated an increase in neural activation may have been responsible for the improvement seen in some sensorimotor measures after the 10-week exercise program. However, in our trial, the physical therapy treatment, which showed an improvement of 12 milliseconds for 2-choice reaction time at 6 weeks, was no different from the improvement seen in the corticosteroid injection or wait-and-see groups. Kauranen ${ }^{37}$ did not include a comparison (control) group in their study, so it is difficult to evaluate the clinical relevance of their findings. It should be noted that the conservative treatments applied in our study were not designed to target sensorimotor function specifically. The treatment interventions used in our study were designed to target pain and functional deficits (eg, pain-free grip strength) rather than sensorimotor deficits. It is possible, therefore, that other treatments that specifically target the sensorimotor deficits may influence measures of sensorimotor function more than was observed in this study.

A post hoc power analysis confirmed that the number of participants in the clinical trial was adequate to identify a clinically important difference over time for the physiotherapy group. Using a data-driven approach in which the standard error of the measure acts as a proxy for the minimal clinically important difference, ${ }^{28}$ an improvement in 2-choice reaction time greater than the standard error of the measure of $17.4 \mathrm{~ms}^{25}$ would require a sample size of 59 participants a group (power $=90 \%, \alpha=.05, \mathrm{SD}=40$ ). Importantly, the sample size of 66 participants a group was based on the previously published outcome measure of global improvement. ${ }^{21}$ This sample size was found to be sufficient to detect statistically and clinically important differences between the treatment groups for measures of global improvement, pain, and function. ${ }^{20}$

Reaction time and speed of movement are only 1 way by which sensorimotor function may be measured. Sensorimotor function also encompasses joint position sense, sensation of force and effort through muscle contraction, and sensations of perceived timing of muscle contractions. ${ }^{38,39}$ Thus, deficits in reaction time and speed of movement may be reflective of deficits in other sensorimotor measures such as joint position error and sensation of force in participants with LE; however, this requires further investigation if we are to understand better the significance of sensorimotor deficits in LE.

A limitation of this study is that the normative data used in this study were measured at a single time point and not repeated over time, as the LE cohort data were. It is possible that the initial improvements seen within the LE cohort represent a learning effect through repeated testing over time. However, if a learning effect is present in sensorimotor testing, then it is reasonable to expect similar improvements over time in a healthy population. As a result, the differences between the participant with LE and the healthy group that we observed in this study may be an underestimation of the real differences. This would add support to our conclusion that sensorimotor deficits remain present over time despite resolution of symptoms.

It is unclear what significance these sensorimotor impairments hold in patients with LE. Several possibilities may explain our findings. One explanation may be that sensorimotor performance is not closely related to pain and disability in the condition of LE, because the change over time is markedly different in sensorimotor measures compared with pain and function measures. ${ }^{20-22}$ If this 
is the case, then it is conceivable that continuing sensorimotor deficits will be present despite reported recovery in patients with LE. It is also feasible that the slower upper-limb reaction times and speed of movement precede the development of chronic injuries such as LE. ${ }^{8,9}$ Finally, the continuation of sensorimotor deficits after a participant reports recovery may explain the tendency of chronic conditions such as LE to recur, ${ }^{40}$ in some ways similar to patellofemoral pain syndrome. ${ }^{41}$ It is clear after our study that further work is required to understand better the significance of sensorimotor impairments in LE.

\section{CONCLUSIONS}

Sensorimotor deficits are bilateral in unilateral LE, and although they demonstrate a tendency toward resolution in the first 6 weeks, deficits were still present after 12 months. The persistence of sensorimotor deficits over time contrasts with the resolution of pain and normalization of function, such as pain-free grip strength. The treatment interventions investigated in this trial did not specifically aim to restore sensorimotor function and did not influence sensorimotor function over time.

Acknowledgments: We acknowledge Ross Darnell, $\mathrm{PhD}$, for assistance with data management and statistical analyses.

\section{References}

1. Allander E. Prevalence, incidence and remission rates of some common rheumatic diseases or syndromes. Scand J Rheumatol 1974;3:145-53.

2. Kivi P. The etiology and conservative treatment of humeral epicondylitis. Scand J Rehabil Med 1983;15:37-41.

3. Kurppa K, Viikari Juntura E, Kuosma E, Huuskonen M, Kivi P. Incidence of tenosynovitis or peritendinitis and epicondylitis in a meat-processing factory. Scand J Work Environ Health 1991;17:32-7.

4. Ranney D, Wells R, Moore A. Upper limb musculoskeletal disorders in highly repetitive industries: precise anatomical physical findings. Ergonomics 1995;38:1408-23.

5. Pienimaki T, Tarvainen T, Siira P, Malmivaara A, Vanharanta H. Associations between pain, grip strength, and manual tests in the treatment evaluation of chronic tennis elbow. Clin J Pain 2002;18:164-70.

6. Sran M, Souvlis T, Vicenzino B, Wright A. Characterisation of chronic lateral epicondylalgia using the McGill pain questionnaire, visual analog scales, and quantitative sensory tests. Pain Clin 2002;13:251-9.

7. Stratford PW, Norman GR, McIntosh JM. Generalizability of grip strength measurements in patients with tennis elbow. Phys Ther 1989;69:276-81.

8. Pienimaki TT, Kauranen K, Vanharanta H. Bilaterally decreased motor performance of arms in patients with chronic tennis elbow. Arch Phys Med Rehabil 1997;78:1092-5.

9. Bisset L, Russell T, Bradley S, Ha B, Vicenzino B. Bilateral sensorimotor abnormalities in unilateral lateral epicondylalgia. Arch Phys Med Rehabil 2006;87:490-5.

10. Lephart S, Riemann B, Fu F. Introduction to the sensorimotor system. In: Lephart S, Fu F, editors. Proprioception and neuromuscular control in joint stability. Champaign: Human Kinetics; 2000. p 37-51.

11. Riemann B, Lephart S. The sensorimotor system, part 1: the physiological basis of functional joint stability. J Athl Train 2002;37:71-7.

12. Cowan SM, Bennell KL, Hodges PW, Crossley KM, McConnell J. Delayed onset of electromyographic activity of vastus medialis obliquus relative to vastus lateralis in subjects with patellofemoral pain syndrome. Arch Phys Med Rehabil 2001;82:183-9. 
13. Hodges P, Moseley G. Pain and motor control of the lumbopelvic region: effect and possible mechanisms. J Electromyogr Kinesiol 2003;13:361-70.

14. Hodges P, Richardson C. Inefficient muscular stabilization of the lumbar spine associated with low back pain: a motor control evaluation of transversus abdominis. Spine 1996;21:2640-50.

15. Barden J, Balyk R, Raso V, Moreau M, Bagnall K. Dynamic upper limb proprioception in multidirectional shoulder instability. Clin Orthop 2004;420:181-9.

16. Myers J, Wassinger C, Lephart S. Sensorimotor contribution to shoulder stability: effect of injury and rehabilitation. Man Ther 2006;11:197-201.

17. Cowan SM, Bennell KL, Crossley KM, Hodges PW, McConnell J. Physical therapy alters recruitment of the vasti in patellofemoral pain syndrome. Med Sci Sports Exerc 2002;34:1879-85. 18. Swanik K, Lephart S, Swanik C, Lephart S, Stone D, Fu F. The effects of shoulder plyometric training on proprioception and selected muscle performance characteristics. J Shoulder Elbow Surg 2002;11:579-86.

19. Crossley K, Bennell K, Green S, Cowan S, McConnell J. Physical therapy for patellofemoral pain: a randomized, double-blinded, placebo-controlled trial. Am J Sports Med 2002;30:857-65.

20. Bisset L, Beller E, Jull G, Brooks P, Darnell R, Vicenzino B. Mobilisation with movement and exercise, corticosteroid injection, or wait and see for tennis elbow: randomised trial. Br Med J 2006;333:939-41.

21. Smidt N, van der Windt DA, Assendelft WJ, Deville WL, Korthals-de Bos IB, Bouter LM. Corticosteroid injections, physiotherapy, or a wait-and-see policy for lateral epicondylitis: a randomised controlled trial. Lancet 2002;359:657-62.

22. Hay E, Paterson S, Lewis M, Hosie G, Croft P. Pragmatic randomised controlled trial of local corticosteroid injection and naproxen for treatment of lateral epicondylitis of elbow in primary care. Br Med J 1999;319:964-8.

23. Mulligan B. Manual therapy - "NAGS", "SNAGS", "MWMS". 4th ed. Wellington: Plane View Services; 1999.

24. Vicenzino B. Lateral epicondylalgia: a musculoskeletal physiotherapy perspective. Man Ther 2003;8:66-79.

25. Kauranen K, Vanharanta H. Influences of aging, gender, and handedness on motor performance of upper and lower extremities. Percept Mot Skills 1996;82:515-25.

26. Kondraske GV. HPM/BEP Manual. 3rd ed. Arlington: Human Performance Measurement Inc; 1991.

27. Stratford PW, Levy DR, Gowland C. Evaluative properties of measures used to assess patients with lateral epicondylitis at the elbow. Physiother Can 1993;45:160-4.

28. Wells G, Beaton D, Shea B, et al. Minimal clinically important differences: review of methods. J Rheumatol 2001;28:406-12.

29. Hodges PW, Moseley GL, Gabrielsson A, Gandevia SC. Experimental muscle pain changes feed forward postural responses of the trunk muscles. Exp Brain Res 2003;151:262-71.

30. Flora H, Braunb C, Elbertc T, Birbaumerc N. Extensive reorganization of primary somatosensory cortex in chronic back pain patients. Neurosci Lett 1997;224:5-8.

31. Abbott JH. Mobilization with movement applied to the elbow affects shoulder range of movement in subjects with lateral epicondylalgia. Man Ther 2001;6:170-7.

32. Kelley J, Lombardo S, Pink M, Perry J, Giangarra C. Electromyographic and cinematographic analysis of elbow function in tennis players with lateral epicondylitis. Am J Sports Med 1994;22:359-63.

33. McCormick K, Zalucki N, Hudson M, Moseley G. Faulty proprioceptive information disrupts motor imagery: an experimental study. Aust J Physiother 2007;53:41-5.

34. McCabe C, Haigh R, Halligan P, Blake D. Simulating sensorymotor incongruence in healthy volunteers: implications for a cortical model of pain. Rheumatology 2005;44:509-16.

35. Bonato C, Zanette G, Manganotti P, et al. "Direct" and "crossed" modulation of human motor cortex excitability following exercise. Neurosci Lett 1993;216:97-100. 
36. Stinear C, Walker K, Byblow W. Symmetric facilitation between motor cortices during contraction of ipsilateral hand muscles. Exp Brain Res 2001;139:101-5.

37. Kauranen KJ, Siira PT, Vanharanta HV. A 10-week strength training program: effect on the motor performance of an unimpaired upper extremity. Arch Phys Med Rehabil 1998;79:925-30. 38. Aydin T, Yildiz Y, Yanmis I, Yildiz C, Kalyon T. Shoulder proprioception: a comparison between the shoulder joint in healthy and surgically repaired shoulders. Arch Orthop Trauma Surg 2001;21:422-5.

39. Gandevia S, McCloskey D, Burke D. Kinaesthetic signals and muscle contraction. Trends Neurosci 1992;15:62-5.

40. Verhaar JAN. Tennis elbow—anatomical, epidemiologic and therapeutic aspects. Int Orthop 1994;18:263-7.

41. Nimon G, Murray D, Sandow M, Goodfellow J. Natural history of anterior knee pain: a 14- to 20-year follow-up of nonoperative management. J Pediatr Orthop 1998;18:118-22.

\section{Supplier}

${ }^{a}$ Sensorimotor Interface Hand Module, Basic Elements of Performance; Human Performance Measurement, 2715 Ave E East, Ste 614, Arlington, TX 76011.

\section{FIGURES}




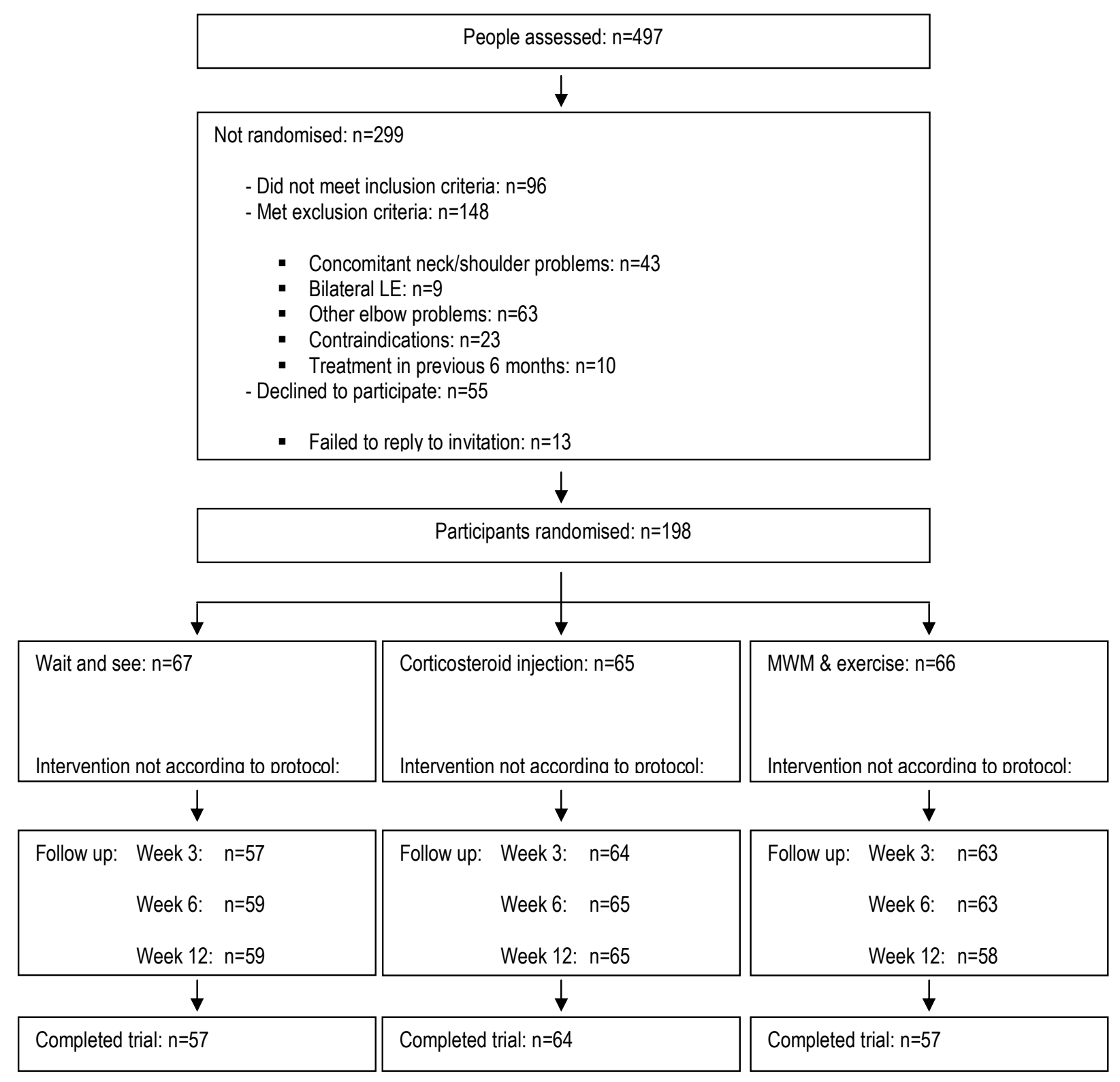

Fig 1. Flow of participants through the study.

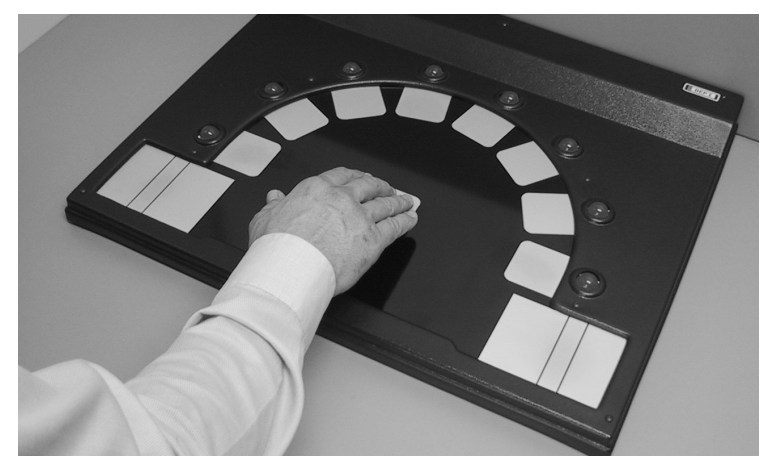

Fig 2. Sensorimotor measures of upper limb reaction time and speed of movement were tested using the Sensorimotor Interface Hand Module of the Human Performance Measurement/Basic Elements of Performance system. ${ }^{\text {a }}$ 


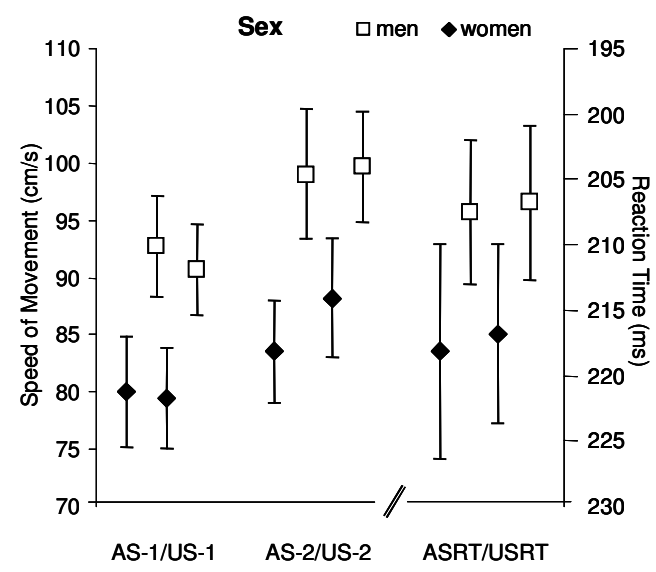

Fig 3. Significant differences in reaction time and speed of movement between men and women at baseline (means and 95\% CIs). Abbreviation: AS, affected speed; US, unaffected speed; ASRT, affected reaction time; USRT, unaffected reaction time.

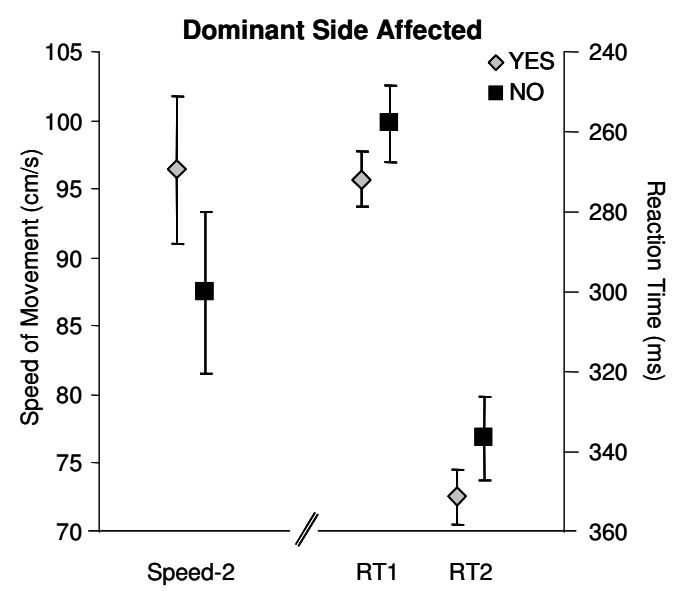

Fig 4. Significant differences in reaction time and speed of movement between dominant and nondominant affected sides at baseline (means and 95\% CIs). Abbreviation: RT, reaction time; Speed-2, speed of movement 2.

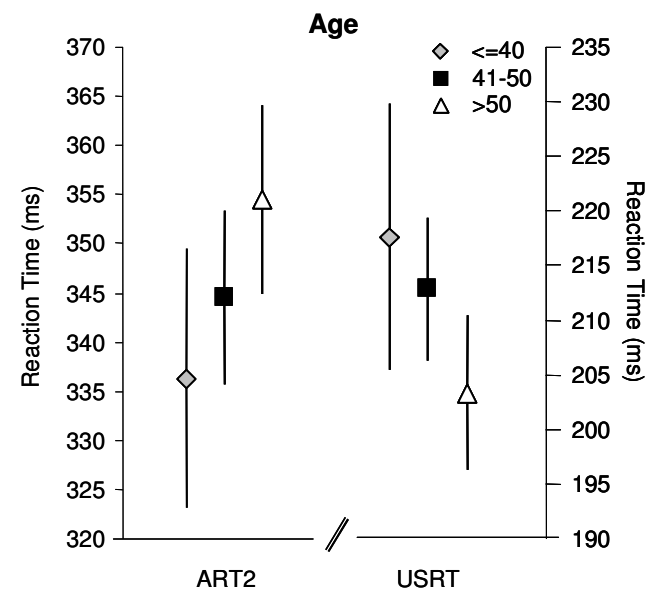

Fig 5. Significant differences in reaction time between age subgroups (decades) at baseline (means and 95\% CIs). Abbreviations: ART2, affected reaction time 2-choice; USRT, unaffected simple 
reaction time.

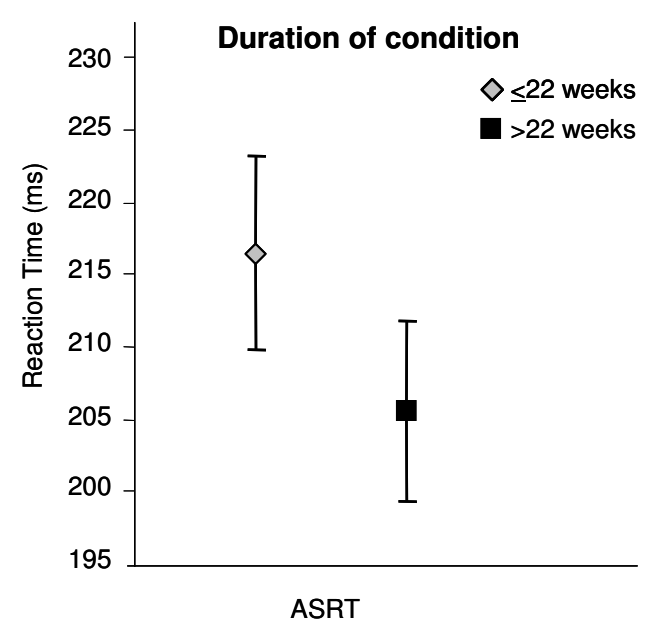

Fig 6. Significant differences in reaction time between participants, subgrouped according to duration of condition at baseline (about the median $=22 \mathrm{wk}$; means and 95\% CIs). Abbreviation: ASRT, affected simple reaction time.

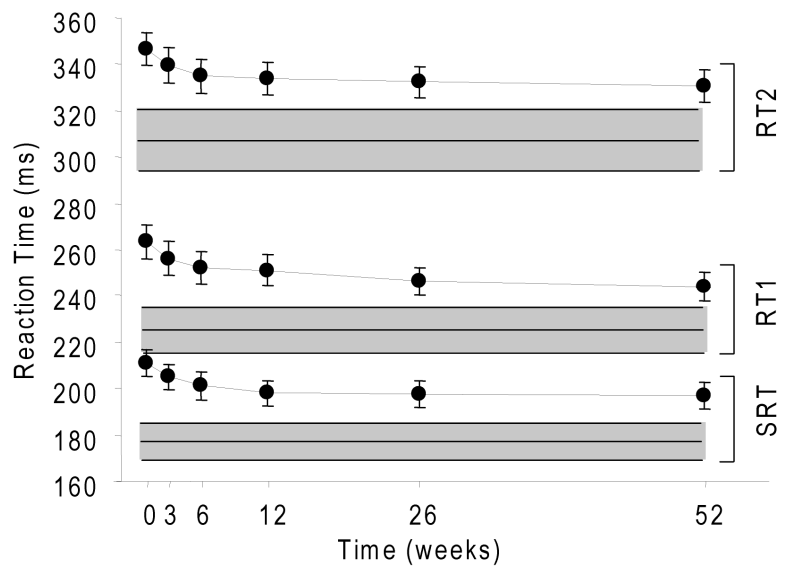

Fig 7. Reaction times for the total LE group over time (means and 99\% CIs) and normative data (means and 99\% CIs) for SRT, 1-choice reaction time (RT1), and 2-choice reaction time (RT2). Normative data (means and $99 \%$ CIs) are represented by the gray bands.

\section{Speed 1}

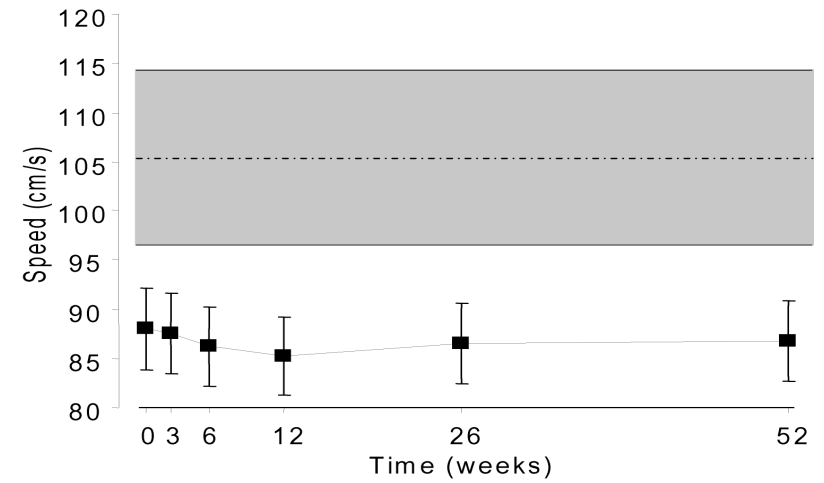

Fig 8. Speed of movement 1 for the total LE group over time (means and 99\% CIs). Normative data (means and $99 \%$ CIs) are represented by the gray bands. 
Speed 2

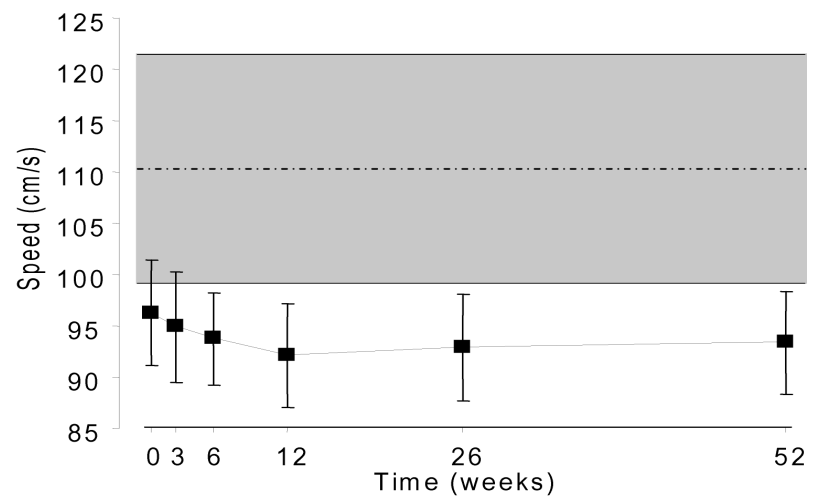

Fig 9. Speed of movement 2 for the total LE group over time (means and 99\% CIs). Normative data (means and $99 \%$ CIs) are represented by the gray bands. 


\section{TABLES}

Table 1: Mean Values \pm SDs for Patient Characteristics at Baseline for the LE Group and Normative Data ${ }^{9}$

\begin{tabular}{|c|c|c|c|}
\hline Baseline Patient Characteristics & $\begin{array}{l}\text { Total LE group } \\
(\mathrm{N}=198)\end{array}$ & & $\begin{array}{l}\text { Normative Data }{ }^{9} \\
(\mathrm{~N}=40)\end{array}$ \\
\hline Age (range) years & $47.6(28-69)$ & & $48.4(33-64)$ \\
\hline Women (\%) & $70(35 \%)$ & & $16(40 \%)$ \\
\hline Dominant side right $(\mathrm{N})$ & 183 & & 36 \\
\hline Mean duration (SD) weeks & $30.9(29.2)$ & & -- \\
\hline \multirow[t]{2}{*}{ Dominant side affected } & 133 & & -- \\
\hline & Affected & Unaffected & Normative data $^{9}$ \\
\hline $\mathrm{SRT}(\mathrm{SD}) \mathrm{ms}$ & $211(33)$ & $210(32)$ & $177(18)$ \\
\hline RT1 (SD) ms & $267(40)$ & $260(43)$ & $225(24)$ \\
\hline $\mathrm{RT} 2$ (SD) ms & $346(41)$ & $344(40)$ & $307(32)$ \\
\hline Speed-1 (SD) cm/s & $88(24)$ & $87(22)$ & $105(21)$ \\
\hline Speed-2 (SD) cm/s & $93(29)$ & $96(26)$ & $110(26)$ \\
\hline
\end{tabular}

Mean SD unless otherwise noted.

Table 2: Mean Difference (99\% CI) for Reaction Time and Speed of Movement at Baseline and 52 Weeks of Follow-Up Between the LE Group and Normative Data

\begin{tabular}{lll}
\hline Sensorimotor measures & Baseline & 52 weeks follow up \\
\hline SRT $\mathrm{ms}$ & $33(24$ to 43$)$ & $20(7$ to 32$)$ \\
RT1 $\mathrm{ms}$ & $39(27$ to 51$)$ & $20(5$ to 34$)$ \\
RT2 $\mathrm{ms}$ & $38(22$ to 54$)$ & $24(7$ to 40$)$ \\
Speed $-1 \mathrm{~cm} / \mathrm{s}$ & $18(8$ to 29$)$ & $19(10$ to 28$)$ \\
Speed-2 cm/s & $16(4$ to 28$)$ & $18(6$ to 29$)$ \\
\hline
\end{tabular}

All measures averaged between sides, SRT = simple reaction time, RT1 = one-choice reaction time, RT2 = two-choice reaction time, Speed-1 = one-choice speed of movement, Speed-2 = two-choice speed of movement. 
Table 3: Mean \pm SD of Each Group, Mean \pm SD Difference Within Each Group, and Mean $\pm 99 \%$ CI Difference Between Groups for Reaction Time and Speed of Movement Outcomes at Primary End Points

\begin{tabular}{|c|c|c|c|c|c|c|c|c|c|c|c|c|}
\hline \multirow[b]{3}{*}{ Outcome } & \multicolumn{12}{|c|}{ Groups } \\
\hline & \multicolumn{4}{|c|}{ Week 0} & \multicolumn{4}{|c|}{ Week 6} & \multicolumn{4}{|c|}{ Week 52} \\
\hline & PT & $\mathrm{Cl}$ & WS & Total & PT & $\mathrm{Cl}$ & WS & Total & PT & $\mathrm{Cl}$ & WS & Total \\
\hline $\mathrm{SRT}{ }^{*}$ affected & $212(29)$ & $208(34)$ & $214(36)$ & $211(33)$ & 196 (31) & $195(32)$ & $206(31)$ & $199(32)$ & $194(32)$ & $192(34)$ & $200(31)$ & $195(32)$ \\
\hline SRT * unaffected & $209(30)$ & $208(30)$ & $214(37)$ & $210(32)$ & $200(29)$ & $203(30)$ & $210(36)$ & $204(32)$ & $195(28)$ & $201(30)$ & $201(36)$ & $199(31)$ \\
\hline RT1 * affected & $263(37)$ & $269(42)$ & $270(40)$ & $267(40)$ & $249(34)$ & $256(49)$ & $265(36)$ & $256(40)$ & $241(29)$ & $243(38)$ & $257(42)$ & 247 (37) \\
\hline RT1 * unaffected & $257(44)$ & $257(39)$ & $264(47)$ & $260(43)$ & $245(32)$ & $246(45)$ & $253(37)$ & $248(38)$ & $237(37)$ & $243(40)$ & $248(35)$ & $243(37)$ \\
\hline RT2 * affected & $346(42)$ & $344(41)$ & $349(42)$ & 346 (41) & $334(36)$ & $335(48)$ & $342(34)$ & $337(40)$ & $333(41)$ & $335(41)$ & $341(40)$ & $337(41)$ \\
\hline RT2 * unaffected & $341(36)$ & $345(43)$ & $347(42)$ & $344(40)$ & $325(34)$ & $327(48)$ & $339(36)$ & $330(40)$ & $325(39)$ & $324(38)$ & $327(40)$ & $325(39)$ \\
\hline Speed-1 Affected & $91(29)$ & $90(25)$ & $84(19)$ & $88(24)$ & $90(25)$ & $88(20)$ & $82(19)$ & $87(22)$ & $91(23)$ & $87(23)$ & $84(19)$ & $88(22)$ \\
\hline Speed-1 unaffected & $90(23)$ & $89(24)$ & $82(19)$ & $87(22)$ & $88(23)$ & $84(19)$ & $81(19)$ & $84(21)$ & $88(22)$ & $85(21)$ & $83(19)$ & $85(21)$ \\
\hline Speed-2 Affected & $96(33)$ & $95(30)$ & $89(24)$ & $93(29)$ & $97(28)$ & $96(24)$ & $86(22)$ & $93(25)$ & $97(27)$ & $94(31)$ & $88(24)$ & $93(28)$ \\
\hline Speed-2 unaffected & $96(26)$ & $98(27)$ & $93(25)$ & $9626)$ & $94(22)$ & $96(24)$ & $90(22)$ & $94(23)$ & $96(25)$ & $94(25)$ & $88(20)$ & $92(24)$ \\
\hline
\end{tabular}

Table 3 continued

\begin{tabular}{|c|c|c|c|c|c|c|c|c|c|c|c|}
\hline \multicolumn{6}{|c|}{ Change within groups } & \multicolumn{6}{|c|}{ Difference between groups } \\
\hline \multicolumn{3}{|c|}{ Week 6 minus Week 0} & \multicolumn{3}{|c|}{ Week 52 minus Week 0} & \multicolumn{3}{|c|}{ Week 6 minus Week 0} & \multicolumn{3}{|c|}{ Week 52 minus Week 0} \\
\hline PT & $\mathrm{Cl}$ & WS & PT & $\mathrm{Cl}$ & WS & PT minus $\mathrm{Cl}$ & PT minus WS & $\mathrm{Cl}$ minus WS & PT minus $\mathrm{Cl}$ & PT minus WS & $\mathrm{Cl}$ minus WS \\
\hline $16(30)$ & $13(29)$ & $7(30)$ & $20(31)$ & $16(27)$ & $16(28)$ & $3(-11$ to 17$)$ & $9(-5$ to 23$)$ & $6(-8$ to 20$)$ & $4(-10$ to 17$)$ & $4(-10$ to 18$)$ & $0(-14$ to 14$)$ \\
\hline $8(29)$ & $5(24)$ & $2(28)$ & $15(30)$ & $8(25)$ & $14(38)$ & $3(-9$ to 15$)$ & $6(-7$ to 19$)$ & $3(-10$ to 16$)$ & $8(-7$ to 22$)$ & $2(-14$ to 17$)$ & $-6(-21$ to 9$)$ \\
\hline $12(43)$ & $12(40)$ & $3(35)$ & $21(37)$ & $26(36)$ & $15(40)$ & $-1(-19$ to 18$)$ & $8(-10$ to 27$)$ & $9(-10$ to 28$)$ & $-4(-22$ to 14$)$ & 7 (-11 to 25$)$ & 11 (-7 to 29$)$ \\
\hline $10(43)$ & $10(43)$ & $13(41)$ & $21(44)$ & $14(33)$ & $19(47)$ & $-1(-20$ to 19$)$ & $-3(-23$ to 17$)$ & $-2(-22$ to 18$)$ & $7(-13$ to 27$)$ & 1 (-19 to 22$)$ & $-6(-25$ to 14$)$ \\
\hline $11(34)$ & $9(34)$ & $9(32)$ & $14(40)$ & $9(33)$ & $11(37)$ & $2(-13$ to 17$)$ & $3(-13$ to 18$)$ & $1(-15$ to 16$)$ & $6(-12$ to 23$)$ & $2(-15$ to 20$)$ & $-3(-20$ to 14$)$ \\
\hline $14(37)$ & $17(36)$ & $11(30)$ & $16(37)$ & $21(30)$ & $24(35)$ & $-3(-19$ to 13$)$ & $3(-13$ to 20$)$ & $6(-10$ to 23$)$ & $-6(-22$ to 11$)$ & $-8(-25$ to 9$)$ & $-2(-19$ to 14$)$ \\
\hline$-2(17)$ & $-2(16)$ & $-4(16)$ & $1(18)$ & $-3(17)$ & $-2(16)$ & $0(-8$ to 7$)$ & $2(-6$ to 10$)$ & $2(-6$ to 10$)$ & $4(-4$ to 12$)$ & $3(-5$ to 11$)$ & $-1(-9$ to 7$)$ \\
\hline$-2(16)$ & $-4(15)$ & $-3(15)$ & $0(16)$ & $-4(16)$ & $-2(16)$ & $2(-5$ to 9$)$ & $2(-6$ to 9$)$ & $-1(-8$ to 6$)$ & $4(-4$ to 11$)$ & $2(-6$ to 9$)$ & $-2(-10$ to 5$)$ \\
\hline $1(18)$ & $0(19)$ & $-6(13)$ & $1(17)$ & $-2(19)$ & $-5(15)$ & $0(-7$ to 8$)$ & $6(-2$ to 14$)$ & $6(-2$ to 14$)$ & $3(-5$ to 12$)$ & $6(-3$ to 14$)$ & $3(-6$ to 11$)$ \\
\hline$-2(18)$ & $-2(17)$ & $-5(18)$ & $0(20)$ & $-5(22)$ & $-9(19)$ & 0 (-9 to 8$)$ & $3(-5$ to 12$)$ & $4(-5$ to 12$)$ & $4(-5$ to 14$)$ & $9(-1$ to 18$)$ & $4(-6$ to 14$)$ \\
\hline
\end{tabular}

